\title{
ON THE LITTLEWOOD-RICHARDSON RULE FOR ALMOST SKEW-SHAPES
}

\author{
GIANDOMENICO BOFFI AND DAVID A. BUCHSBAUM \\ (Communicated by Bernd Ulrich)
}

\begin{abstract}
We describe combinatorially the coefficients occurring in the irreducible decomposition of the Weyl module associated with an almost skewshape belonging to the family $J$.

The proof uses the fundamental exact sequence for almost skew-shapes to initiate an inductive procedure which ultimately reduces to the classical Littlewood-Richardson rule for skew partitions.
\end{abstract}

\section{INTRODUCTION}

The Weyl module, $K_{\lambda / \mu}(F)$, associated with a skew-partition $\lambda / \mu$ is a representation of the general linear group $G L(F)$, where $F$ stands for a finite free $R$ module. Hence, if $R$ is a field of characteristic zero, $K_{\lambda / \mu}(F)$ is isomorphic to a direct sum of Weyl modules, $K_{\nu}(F)$, associated with ordinary partitions $\nu$. For under that assumption on $R$, it is well known (see, for instance, [3], Chapter I) that every finite dimensional representation, such as $K_{\lambda / \mu}(F)$, is completely reducible, and a complete set of irreducibles is given by the modules $K_{\nu}(F)$. The classical Littlewood-Richardson rule describes the partitions $\nu$ occurring in the isomorphism:

$$
K_{\lambda / \mu}(F) \cong \bigoplus_{\nu} g(\lambda / \mu ; \nu) K_{\nu} F
$$

Namely,

Theorem 1.1 (Cf. [3], Chapter I; in particular, Section 9). $g(\lambda / \mu ; \nu)$ is the number of ways (possibly zero) in which we can fill the diagram of the skew-partition $\lambda / \nu$ with all the elements of the set of integers

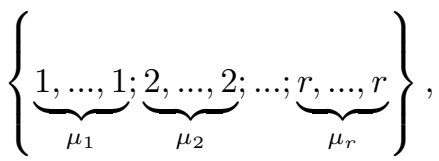

$r=\operatorname{length}(\mu)$, so that

(a) the resulting tableau $T$ is (Weyl-) standard, that is, each row of $T$ is nondecreasing and each column is strictly increasing,

Received by the editors September 4, 2006.

2000 Mathematics Subject Classification. Primary 05E10, 20G05; Secondary 13D25.

Key words and phrases. Almost skew-shape, Weyl module, Littlewood-Richardson rule.

The first author was partially supported by MIUR and is a member of GNSAGA - INdAM. 
(b) the word associated with $T$, as $(T)$, obtained by listing all entries of $T$ from right to left on each row, starting from the top row, is a lattice permutation.

Now let $\lambda / \mu$ stand for an almost skew-shape, where $\lambda=\left(\lambda_{1}, \ldots, \lambda_{n}\right)$ is a partition of length $n$ and $\mu$ is a sequence of integers $\left(\mu_{1}, \ldots, \mu_{n}\right)$ such that

$$
\lambda_{i} \geq \mu_{i} \forall i=1, \ldots, n, \quad \mu_{1} \geq \ldots \geq \mu_{n-1} \quad \text { and } \quad 0 \leq \mu_{n} \leq \mu_{1} .
$$

In other words, the almost skew-shape is a skew-partition but for its last row, which, rather than projecting beyond (or flush with) the penultimate row, may not make it that far on the left.

We define the type, $\tau$, of the given almost skew-shape as the integer $n-(i+1)$, where $i$ is the largest index different from $n$ such that $\mu_{n} \leq \mu_{i}$. Thus $\tau=0$ $(i=n-1)$ means that the almost skew-shape is in fact a skew-partition, while $\tau>0(i \leq n-2)$ means that the last row is actually indented on the left from the penultimate row.

Since different pairs $(\lambda, \mu)$ may yield the same almost skew-shape, in order to have a canonical description of our almost skew-shapes of positive type, from now on we assume that $\mu_{n-1}=0$ whenever $\tau>0$. In particular, we have

$$
\mu_{1} \geq \ldots \geq \mu_{i} \geq \mu_{n} \gtreqless \mu_{i+1} \geq \mu_{i+2} \geq \ldots \geq \mu_{n-2} \geq \mu_{n-1}=0 .
$$

We call $\mu^{\prime}$ the partition $\left(\mu_{1}, \ldots, \mu_{i}, \mu_{n}, \mu_{i+1}, \mu_{i+2}, \ldots, \mu_{n-2}\right)$; it has length at most $n-1$.

For more details about almost skew-shapes, and Weyl modules associated with them, see [2], Chapter VI.

If $K_{\lambda / \mu}(F)$ denotes the Weyl module associated with an almost skew-shape $\lambda / \mu$ of positive type, again we have in characteristic zero an isomorphism

$$
K_{\lambda / \mu}(F) \cong \bigoplus_{\nu} h(\lambda / \mu ; \nu) K_{\nu} F
$$

It is the purpose ot this note to describe for the first time the coefficients $h(\lambda / \mu ; \nu)$. Namely,

Theorem 1.2. Assume that $\lambda_{n-1}-\lambda_{n} \geq \tau(>0)$. Then $h(\lambda / \mu ; \nu)$ is the number of ways (possibly zero) in which we can fill the diagram of the skew-partition $\lambda / \nu$ with all the elements of the set of integers

$$
\{\underbrace{1, \ldots, 1}_{\mu_{1}^{\prime}} ; \underbrace{2, \ldots, 2}_{\mu_{2}^{\prime}} ; \ldots ; \underbrace{r, \ldots, r}_{\mu_{r}^{\prime}}\},
$$

$r=\operatorname{length}\left(\mu^{\prime}\right)$, so that

(a) the resulting tableau $T$ is (Weyl-) standard,

(b) the word associated with $T$, as $(T)$, is a lattice permutation,

(c) if $k$ is the largest index occurring in $T$ ( $k \geq i+1=n-\tau$ for sure, since $\left.\mu_{n}>0\right)$, then $k$ only occurs on the $n$-th row of $\lambda$; furthermore, if $k>n-\tau$, then the number of times $n-\tau$ occurs on the first $n-1$ rows of $\lambda$ equals the number of times $k$ occurs on the $n$-th row. 
One should remark that



The rest of this paper is devoted to a proof of Theorem 1.2. As also indicated by some examples, we suspect that the assumption $\lambda_{n-1}-\lambda_{n} \geq \tau$ can be removed. But it is necessary for our inductive proof.

Consistent with [1], we will say that an almost skew-shape $\lambda / \mu$ of type $\tau$ "belongs to the family $J$ " whenever $\lambda_{n-1}-\lambda_{n} \geq \tau$. In particular, all skew-partitions belong to the family $J$.

\section{Proof of Theorem 1.2: Outline and preparations}

Our inductive proof of Theorem 1.2 is based on the fundamental short exact sequence for almost skew-shapes, which is Theorem VII.1.2 of [2] (a theorem dealing with Weyl-Schur complexes, not just with modules). More precisely, thanks to the assumption $\lambda_{n-1}-\lambda_{n} \geq \tau$, we can recover our $K_{\lambda / \mu}(F)$ as the leftmost term of a suitable instance of that fundamental short exact sequence. Since the central and rightmost terms have lower type and still belong to the family $J$, their decompositions into irreducibles are known by induction and the exactness of the sequence yields the decomposition of $K_{\lambda / \mu}(F)$.

Proposition 2.1 (Special instance of the fundamental exact sequence). Notation as above and $\tau>0$. Then there is a short exact sequence

$$
0 \rightarrow K_{\lambda / \mu}(F) \rightarrow K_{\widehat{\lambda} / \widehat{\mu}}(F) \rightarrow K_{\bar{\lambda} / \bar{\mu}}(F) \rightarrow 0
$$

where:

$$
\begin{aligned}
& \widehat{\lambda}=\lambda, \\
& \widehat{\mu}=\left(\mu_{1}, \ldots, \mu_{i}, \mu_{n}, \mu_{i+2}, \ldots, \mu_{n-2}, \mu_{n-1}=0, \mu_{i+1}\right)
\end{aligned}
$$

[if $i$ is maximal, i.e. $i+1=n-1$, then $\left.\widehat{\mu}=\left(\mu_{1}, \ldots, \mu_{i}, \mu_{n}\right)\right]$,

$\bar{\lambda}=\left(\lambda_{1}, \ldots, \lambda_{n-1}, \lambda_{n}+1\right)$,

$\bar{\mu}=\left(\mu_{1}, \ldots, \mu_{i}, \mu_{n}, \mu_{i+2}, \ldots, \mu_{n-2}, \mu_{n-1}=0, \mu_{i+1}+1\right)$

[if $i$ is maximal, then $\left.\bar{\mu}=\left(\mu_{1}, \ldots, \mu_{i}, \mu_{n}, 1\right)\right]$.

We call $\widehat{\tau}$ and $\bar{\tau}$ the types of $\widehat{\lambda} / \widehat{\mu}$ and $\bar{\lambda} / \bar{\mu}$, respectively.

It is an easy remark that $\widehat{\tau} \lesseqgtr \tau$, and hence $\widehat{\lambda} / \widehat{\mu}$ still belongs to the family $J$. More precisely, let $u$ be the positive integer such that

$$
\mu_{1} \geq \ldots \geq \mu_{i} \geq \mu_{n} \gtreqless \mu_{i+1}=\mu_{i+2}=\ldots=\mu_{i+u} \gtreqless \mu_{i+u+1} \geq \ldots \geq \mu_{n-2} \geq \mu_{n-1} ;
$$

then $\widehat{\tau}=\tau-u$.

As for $\bar{\tau}$, since

$$
\mu_{1} \geq \ldots \geq \mu_{i} \geq \mu_{n} \geq \mu_{i+1}+1 \supsetneqq \mu_{i+2} \geq \ldots \geq \mu_{n-2} \geq \mu_{n-1}=0,
$$


we always get $\bar{\tau}=\tau-1$. It also follows that $\bar{\lambda} / \bar{\mu}$ still belongs to the family $J$, because $\bar{\lambda}$ is obtained from $\lambda$ by adding an extra box at the rightmost end of the last row.

Summarizing, the short exact sequence of Proposition 2.1 does not bring us outside of the family $J$, and Theorems 1.1 and 1.2 (the latter by induction hypothesis) apply to $\widehat{\lambda} / \widehat{\mu}$ and $\bar{\lambda} / \bar{\mu}$.

Three cases will have to be examined:

(1) $\widehat{\tau}=0=\bar{\tau}$ (which is equivalent to $\tau=1$ ),

(2) $\widehat{\tau}=0$ and $\bar{\tau}>0$ (with $\tau=u=\bar{\tau}+1$ ),

(3) $0<\widehat{\tau}=\tau-u \leq \tau-1=\bar{\tau}$.

Before going into the details, we need some notation. Given a skew-partition $\lambda / \nu$, and a tableau, $T$, of that shape, we denote by $C_{\ell}^{T}(s)$ (respectively, $C^{T}(s)$ ) the number of times the index $s$ occurs in $T$ on the $\ell$-th row of $\lambda$ (respectively, on all rows of $\lambda$ ). The letter $C$ is meant to recall the word "content."

With this notation, condition (c) of Theorem 1.2 reads as follows: $C^{T}(k)=$ $C_{n}^{T}(k)$ always, and also $C^{T}(n-\tau)-C_{n}^{T}(n-\tau)=C^{T}(k)$, whenever $k>n-\tau$.

\section{Proof of Theorem 1.2: Details}

3.1. Case 1: $\widehat{\tau}=0=\bar{\tau}$ (which is equivalent to $\tau=1$ ).

The numbers occurring in the set

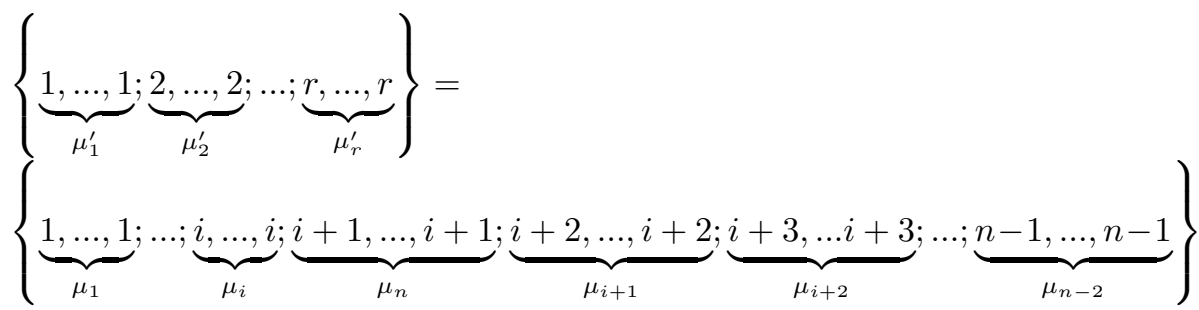

of Theorem 1.2 can also be obtained by rearranging the parts of $\widehat{\mu}$ in order to get a partition, say $\widehat{\mu}^{\prime}$, and then taking $\widehat{\mu}_{1}^{\prime}$ copies of $1, \widehat{\mu}_{2}^{\prime}$ copies of 2 , etc.

By Theorem 1.1, it follows that $g(\widehat{\lambda} / \widehat{\mu} ; \nu)$ is parametrized by the tableaux $T$ of shape $\hat{\lambda} / \nu=\lambda / \nu$ such that

- $T$ is filled with the indices $\underbrace{1, \ldots, 1}_{\mu_{1}^{\prime}} ; \underbrace{2, \ldots, 2}_{\mu_{2}^{\prime}} ; \ldots ; \underbrace{r, \ldots, r}_{\mu_{r}^{\prime}}$,

- $T$ is standard and $a s(T)$ is a lattice permutation.

Hence $h(\lambda / \mu ; \nu)=g(\widehat{\lambda} / \widehat{\mu} ; \nu)-g(\bar{\lambda} / \bar{\mu} ; \nu)$ will be parametrized by the previous tableaux $T$ which cannot be put in one-to-one correspondence with the tableaux $\bar{T}$ parametrizing $g(\bar{\lambda} / \bar{\mu} ; \nu)$. These tableaux $\bar{T}$ have the following properties:

- the shape is that of $T$, but with the addition of an extra box at the rightmost end of the $n$-th row of $\lambda$,

- the indices filling $\bar{T}$ are the same as $T$, but with the addition of a further index $n$,

- $\bar{T}$ is standard and $a s(\bar{T})$ is a lattice permutation.

Since $\bar{T}$ is standard, its index $n$ must occur on the $n$-th row of $\lambda$, at the rightmost end. Thus if we remove from $\bar{T}$ the box containing $n$, a tableau $T$ related to $g(\widehat{\lambda} / \widehat{\mu} ; \nu)$ is obtained. Conversely, taking a tableau $T$ related to $g(\widehat{\lambda} / \widehat{\mu} ; \nu)$ and adding an extra box, containing $n$, to the rightmost end of the $n$-th row of $\lambda$, 
we always get a tableau $\bar{T}$ related to $g(\bar{\lambda} / \bar{\mu} ; \nu)$, provided $a s(\bar{T})$ stays a lattice permutation. But as $(\bar{T})$ fails to be a lattice permutation precisely when $C^{T}(n-1)=$ $C_{n}^{T}(n-1)$. Hence the tableaux $T$ related to $h(\lambda / \mu ; \nu)$ have the following properties:

- $T$ is of shape $\lambda / \nu$,

- $T$ is filled with the indices $\underbrace{1, \ldots, 1}_{\mu_{1}^{\prime}} ; \underbrace{2, \ldots, 2}_{\mu_{2}^{\prime}} ; \ldots ; \underbrace{r, \ldots, r}_{\mu_{r}^{\prime}}$,

- $T$ is standard and $a s(T)$ is a lattice permutation,

- the largest index occurring in $T$ only occurs on the $n$-th row of $\lambda$.

Since the largest index is $n-1=n-\tau$, condition (c) of Theorem 1.2 is fully satisfied and Case 1 is completely proved.

Remark 3.1. In the above, the skew-partition $\bar{\lambda} / \bar{\mu}$ has been represented in a rather unusual form, since $\bar{\mu}_{n}=1$.

3.2. Case 2: $\widehat{\tau}=0$ and $\bar{\tau}>0$ (with $\tau=u=\bar{\tau}+1$ ).

As in Case $1, g(\widehat{\lambda} / \widehat{\mu} ; \nu)$ is parametrized by the tableaux $T$ of shape $\widehat{\lambda} / \nu=\lambda / \nu$ such that

- $T$ is filled with the indices $\underbrace{1, \ldots, 1}_{\mu_{1}^{\prime}} ; \underbrace{2, \ldots, 2}_{\mu_{2}^{\prime}} ; \ldots ; \underbrace{r, \ldots, r}_{\mu_{r}^{\prime}}$,

- $T$ is standard and $a s(T)$ is a lattice permutation.

We point out to the reader that the indices occurring in $T$ never exceed $n-\tau$, since $\tau=u$.

By Theorem 1.2, the tableaux $\bar{T}$ related to $h(\bar{\lambda} / \bar{\mu} ; \nu)$ have the following properties:

- the shape is that of $T$, but with the addition of an extra box at the rightmost end of the $n$-th row of $\lambda$,

- the indices filling $\bar{T}$ are the same as $T$, but with the addition of a further index $n-\tau+1$,

- $\bar{T}$ is standard and $a s(\bar{T})$ is a lattice permutation,

- $n-\tau+1$ occurs on the $n$-th row of $\bar{\lambda}$.

Clearly, taking a tableau $T$ related to $g(\widehat{\lambda} / \widehat{\mu} ; \nu)$ and adding an extra box (filled with $n-\tau+1)$ to the rightmost end of the $n$-th row of $\lambda$, one gets a tableau $\bar{T}$ related to $h(\bar{\lambda} / \bar{\mu} ; \nu)$, provided $a s(\bar{T})$ stays a lattice permutation. This fails to happen when $C^{T}(n-\tau)=C_{n}^{T}(n-\tau)$. Hence the tableaux $T$ related to $h(\lambda / \mu ; \nu)$ have the following properties:

- $T$ is of shape $\lambda / \nu$,

- $T$ is filled with the indices $\underbrace{1, \ldots, 1}_{\mu_{1}^{\prime}} ; \underbrace{2, \ldots, 2}_{\mu_{2}^{\prime}} ; \ldots ; \underbrace{r, \ldots, r}_{\mu_{r}^{\prime}}$,

- $T$ is standard and $a s(T)$ is a lattice permutation,

- the largest index occurring in $T$ only occurs on the $n$-th row of $\lambda$.

Since the largest index is $n-\tau$, condition (c) of Theorem 1.2 is fully satisfied and Case 2 is completely proved. 
3.3. Case 3: $0<\widehat{\tau}=\tau-u \leq \tau-1=\bar{\tau}$.

By Theorem 1.2, $h(\widehat{\lambda} / \widehat{\mu} ; \nu)$ is parametrized by the tableaux $T$ of shape $\widehat{\lambda} / \nu=\lambda / \nu$ such that

- $T$ is filled with the indices $\underbrace{1, \ldots, 1}_{\mu_{1}^{\prime}} ; \underbrace{2, \ldots, 2}_{\mu_{2}^{\prime}} ; \ldots ; \underbrace{r, \ldots, r}_{\mu_{r}^{\prime}}$,

- $T$ is standard and $a s(T)$ is a lattice permutation,

- the largest index $k=i+1+u=n-\tau+u=n-\widehat{\tau}$ only occurs on the $n$-th row of $\lambda$.

The tableaux $\bar{T}$ related to $h(\bar{\lambda} / \bar{\mu} ; \nu)$ have the following properties:

- the shape is that of $T$, but with the addition of an extra box at the rightmost end of the $n$-th row of $\lambda$,

- the indices filling $\bar{T}$ are the same as $T$, but with the addition of a further index $n-\tau+1=n-\bar{\tau}$,

- $\bar{T}$ is standard and $a s(\bar{T})$ is a lattice permutation,

- the largest index $k=n-\tau+u$ only occurs on the $n$-th row of $\bar{\lambda}$ and, if $u \geq 2$,

$$
C^{\bar{T}}(n-\tau+1)-C_{n}^{\bar{T}}(n-\tau+1)=C^{\bar{T}}(n-\tau+u) .
$$

If $u=1$, it is obvious that if we remove from $\bar{T}$ the rightmost box of the last row (a box filled with the largest index $n-\tau+1$ ), we obtain a tableau $T$ related to $h(\widehat{\lambda} / \widehat{\mu} ; \nu)$. If $u \geq 2$, then condition $(*)$ says that there is a copy of $n-\tau+1$ (no longer the largest index) on the $n$-th row of $\bar{\lambda}$. Call $\overline{\bar{T}}$ the tableau obtained from $\bar{T}$ by bringing that $n-\tau+1$ into the rightmost box of the same row. If we remove from $\overline{\bar{T}}$ that rightmost box, again we get a tableau $T$ related to $h(\widehat{\lambda} / \widehat{\mu} ; \nu)$.

Conversely, taking a tableau $T$ related to $h(\widehat{\lambda} / \widehat{\mu} ; \nu)$ and adding to the right end of the $n$-th row of $\lambda$ a new box containing $n-\tau+1$, we obtain a tableau $\bar{T}$ which may or may not be related to $h(\bar{\lambda} / \bar{\mu} ; \nu)$. However, since

$$
\mu_{i+2}^{\prime}=\ldots=\mu_{i+1+u}^{\prime},
$$

if we call $\overline{\bar{T}}$ the tableau obtained by rearranging the last row of $\bar{T}$ in increasing order, the new index $n-\tau+1$ does not have larger indices on top of it in $\overline{\bar{T}}$, and the latter is therefore standard. As for $a s(\overline{\bar{T}})$ being a lattice permutation, this amounts to asking whether the following condition held for $T$ :

$$
C^{T}(n-\tau)-C_{n}^{T}(n-\tau) \gtreqless C^{T}(n-\tau+1)-C_{n}^{T}(n-\tau+1) .
$$

It follows from the above that a tableau $T$ related to $h(\widehat{\lambda} / \widehat{\mu} ; \nu)$ is in fact related to $h(\lambda / \mu ; \nu)$ if, in addition, it satisfies

$$
C^{T}(n-\tau)-C_{n}^{T}(n-\tau)=C^{T}(n-\tau+1)-C_{n}^{T}(n-\tau+1) .
$$

Recall, though, that $\mu_{i+2}^{\prime}=\ldots=\mu_{i+1+u}^{\prime}$ means

$$
C^{T}(n-\tau+1)=\ldots=C^{T}(n-\tau+u),
$$

so that $C_{n}^{T}(n-\tau+1)=0$ because $a s(T)$ is a lattice permutation and $n-\tau+u$ only occurs on the $n$-th row of $\lambda$. Hence $(* *)$ translates into

$$
C^{T}(n-\tau)-C_{n}^{T}(n-\tau)=C^{T}(n-\tau+1),
$$


that is $($ by $(\#))$, into

$$
C^{T}(n-\tau)-C_{n}^{T}(n-\tau)=C^{T}(n-\tau+u),
$$

as required.

This completes the proof of Case 3, as well as of Theorem 1.2.

\section{REFERENCES}

[1] K. Akin and D. A. Buchsbaum, Characteristic-free representation theory of the general linear group, 2. Homological considerations, Adv. Math. 72 (1988), 171-210. MR972760 (90e:20037)

[2] G. Boffi and D. A. Buchsbaum, Threading Homology Through Algebra: Selected Patterns, Oxford University Press (Clarendon), Oxford 2006. MR2247272 (2007g:13018)

[3] I. G. Macdonald, Symmetric Functions and Hall Polynomials, Oxford University Press (Clarendon), Oxford 1979. MR553598 (84g:05003)

Dipartimento di Scienze, Università "G. D’Annunzio", Viale Pindaro 42, 65127 PesCARA, ITALY

E-mail address: gboffi@unich.it

Department of Mathematics, Brandeis University, Waltham, Massachusetts 02254

E-mail address: buchsbau@brandeis.edu 